\title{
Efeitos da Adubação Nitrogenada e de Sistemas de Manejo sobre a Morfogênese de Lolium multiflorum Lam. e Paspalum urvillei Steud. em Ambiente de Várzea
}

\author{
Fernando Luiz Ferreira de Quadros ${ }^{1}$, Duilio Guerra Bandinelli 2
}

\begin{abstract}
RESUMO - Objetivou-se, com este experimento, avaliar a resposta morfogênica de Lolium multiflorum Lam. e Paspalum urvillei Steud., submetidos a níveis de adubação nitrogenada (100, 200 e $300 \mathrm{~kg} / \mathrm{ha}$ de N). Os novilhos mantidos nesta pastagem recebiam diferentes níveis de suplementação energética $(0,0 ; 0,4$ e $0,8 \%$ do peso vivo), respectivamente, para os tratamentos de adubação. Adotou-se delineamento inteiramente casualizado, sendo avaliados 90 afilhos por tratamento, 45 de cada espécie por repetição. Foram utilizados afilhos marcados para avaliar as taxas de alongamento, aparecimento e desfolha e o filocrono de lâminas foliares das espécies. A suplementação propiciou efeito substitutivo para L. multiflorum, com menores taxas de desfolha no tratamento $300 \mathrm{~kg} / \mathrm{ha}$ de $\mathrm{N}+0,8 \%$ PV. Em P. urvillei, a taxa de desfolha foi elevada, independentemente de sua contribuição na massa de forragem. As maiores taxas de alongamento de L. multiflorum foram observadas com o uso de $300 \mathrm{~kg} / \mathrm{ha} \mathrm{de} \mathrm{N}$, o que induz à aplicação de maiores doses de $\mathrm{N}$ nos estádios iniciais. No período inicial, registrou-se a maior taxa de aparecimento de folhas no tratamento $100 \mathrm{~kg} /$ ha de N, com 12,5 dias para o aparecimento de nova folha. No segundo período, foram semelhantes e, no terceiro, o tratamento com $200 \mathrm{~kg} / \mathrm{h}$ a de $\mathrm{N}$ apresentou a maior taxa, sendo necessários, em média, 14,1 dias para surgir nova folha. Com a aproximação do final do ciclo da espécie, a taxa de aparecimento diminuiu. A adubação nitrogenada não interfere no filocrono de ambas as espécies. A taxa de alongamento foliar de $P$. urvillei no tratamento com $300 \mathrm{~kg} / \mathrm{ha}$ de $\mathrm{N}$ foi superior ao menor nível de $\mathrm{N}$, em todos os períodos A taxa média de aparecimento de folhas de $P$. urvillei foi de 0,07 folhas/dia. O intervalo médio para o aparecimento de nova folha foi de 14,1 dias, sendo influenciada principalmente pela soma térmica acumulada.
\end{abstract}

Palavras-chave: afilhos marcados, filocrono, taxa de alongamento de folhas, taxa de aparecimento de folhas, taxa de desfolha

\section{Effects of Nitrogen Fertilization and Management Systems on Morphogenesis of Lolium multiflorum Lam. and Paspalum urvillei Steud. in a Lowland Area}

\begin{abstract}
The objective of this trial was to evaluate morphogenic response of Lolium multiflorum Lam. and Paspalum urvillei Steud., under levels of nitrogen fertilization $(100,200$ and $300 \mathrm{~kg} / \mathrm{ha}$ of N). Steers grazing this pasture received different energy levels supplementation $(0.0,0.4$ and $0.8 \%$ of live weight [LW]), respectively, for the fertilization levels. A completely randomized design was used, where 90 tillers were evaluated by treatment ( 45 of each specie by replicate). Marked tillers were used to evaluate elongation, appearance and defoliation rates and the phyllochron of leaf laminas of the species. Supplementation provided a substitutive effect for $L$. multiflorum, with lower defoliation rates in the $300 \mathrm{~kg} / \mathrm{ha}$ of $\mathrm{N}+0.8 \% \mathrm{LW}$ treatment. For $P$. urvillei, the defoliation rate was higher, independently of its contribution in forage mass. The higher elongation rates of L. multiflorum was observed with $300 \mathrm{~kg} / \mathrm{ha}$ of N, motivates the application of higher dosis in the earlier stages. In the first period, it was observed the higher leaf appearance rate for the $100 \mathrm{~kg} / \mathrm{ha}$ of $\mathrm{N}$ treatment, with 12.5 days for the appearance of a new leaf. In the second one, the rates was similar and, in the third one, $200 \mathrm{~kg} / \mathrm{ha}$ of $\mathrm{N}$ treatment presented the higher rate, being necessary 14.1 days, in average, for a new leaf to rise. As the end of the growing season is reached, the appearance rate was shortened. Nitrogen fertilization didn't affected phyllochron of both species. The elongation rate of $P$. urville $i$ in $300 \mathrm{~kg} / \mathrm{ha}$ of $\mathrm{N}$ treatment was higher than the lower level of $\mathrm{N}$ in all periods. The leaf appearance rate of $P$. urvillei was of 0.07 leaves/ day. The average interval for a new leaf to appear was of 14.1 days, being affected by the accumulated thermal total amount.
\end{abstract}

Key Words: marked tillers, phyllochron, leaf elongation rate, leaf appearance rate, defoliation rate

\section{Introdução}

No estado do Rio Grande do Sul, estima-se que cerca de 650 mil hectares de áreas cultivadas com arroz irrigado permanecem, anualmente, em pousio. A utilização das áreas de várzea, com espécies forrageiras de estação fria, visam aumentar a produtividade do sistema de produção agro-pastoril, principalmente no período em que estas áreas ficam em pousio. Para utilização mais rentável e intensiva das áreas de várzea durante o inverno, Nabinger (1986) e Marchezan et al. (1998) sugerem como alternativa

\footnotetext{
${ }^{1}$ Engenheiro-Agrônomo, Professor Doutor do Departamento de Zootecnia. Campus da UFSM, Santa Maria, RS, Brasil. CEP: 97105-900 E.mail: fquadros@ccr.ufsm.br. Autor para correspondência.

2 Engenheiro-Agrônomo, Acadêmico do curso de Pós-Graduação em Zootecnia da UFSM-RS, Bolsista CAPES. E.mail: bandlli@zipmail.com.br
} 
o uso de pastagens cultivadas, principalmente à consorciação de gramíneas e leguminosas, desde que as espécies sejam adaptadas ao ambiente úmido e sob manejo adequado. Alguns estudos já foram realizados com o objetivo de verificar a adaptação de plantas forrageiras introduzidas no ambiente de várzea, destacando-se, entre as gramíneas, o azevém (Lolium multiflorum Lam.) (Marchezan et al., 1998). Neste ambiente mal drenado, uma das principais espécies nativas que contribuem em produção de matéria seca (MS), que se desenvolve na flora de sucessão, é Paspalum urvillei Steud. (Bandinelli et al., 2000).

A morfogênese de plantas determina o fluxo de novos tecidos, tendo o C (carbono) e o N (nitrogênio) como elementos indispensáveis no processo. Esta pode ser definida ao nível de planta individual ou em unidade de crescimento autotrófico, como o afilho para as gramíneas e o estolão enraizado para o trevo branco (Trifolium repens L.) (Lemaire \& Chapman, 1996).

O nitrogênio tem importante papel na morfogênese de plantas pela ação diferencial nas variáveis morfogênicas determinantes da estrutura da pastagem, podendo propiciar às plantas condições de aumentar as taxas de expansão de folhas e de afilhamento, com ligeiro efeito na taxa de aparecimento de folhas (Cruz \& Boval, 1999).

O manejo de pastagens consiste na tomada de decisões técnicas capazes de manter o equilíbrio entre dois fatores conflitantes de produção: a exigência do animal sob pastejo e a exigência fisiológica da planta forrageira para alcançar e manter elevada produtividade (Corsi \& Nascimento Jr., 1994). Entre as alternativas de manejo dstaca-se o uso de suplementação, que pode promover quatro tipos de interações entre a pastagem, o suplemento e o desempenho animal: efeito aditivo do suplemento sobre o consumo da pastagem; efeito aditivo do suplemento sobre a produção; efeito substitutivo do suplemento sobre o consumo do pasto sem alteração do ganho individual e; efeito substitutivo do suplemento sobre o consumo de pasto, com alteração de ganho individual (Rocha, 1998).

O regime ou padrão de desfolhação - variável de maior influência na resposta da planta ao pastejo pode ser definida pela intensidade, pela quantidade de material da planta removido e frequiência pelo número desfolhas da planta em determinando período de tempo de desfolhação (Gillen et al., 1990).

A desfolhação determina modificações estruturais na vegetação que se expressa pela redução da superfície foliar, afetando o fornecimento de assimi- lados que são requeridos para sustentar as funções de crescimento de órgãos aéreos e subterrâneos e, eventualmente, no número de meristemas de crescimento (Mazzanti, 1997).

Em razão da importância das espécies L. multiflorum e P. urvillei para as áreas de várzea, é necessário o conhecimento de aspectos referentes à morfogênese destas plantas, neste ambiente, frente a diferentes alternativas de manejo. O presente estudo foi conduzido com o objetivo de avaliar a influência de diferentes níveis de adubação nitrogenada nas variáveis morfogênicas: taxas de alongamento e aparecimento de lâminas foliares, determinando valores de filocrono e o efeito da suplementação nas taxas de desfolha das espécies L. multiflorum e P. urvillei.

\section{Material e Métodos}

O estudo foi conduzido em área de várzea, pertencente ao Departamento de Fitotecnia da UFSMRS, localizada na região fisiográfica denominada Depressão Central, do RS, com coordenadas de $29^{\circ} 43^{\prime}$ de latitude Sul e $53^{\circ} 42^{\prime}$ de longitude a Oeste de Greenwich. O clima da região é subtropical úmido (Cfa), com temperatura e precipitação pluviométrica médias anuais de $19,2^{\circ} \mathrm{Ce} 1.769 \mathrm{~mm}$, respectivamente. O solo é classificado segundo o sistema brasileiro como Planossolo Hidromórfico Eutrófico arênico (Embrapa, 1999).

A área sofreu processo de sistematização com desnível de 0,06\%, após o cultivo de arroz irrigado, em 1997. Após a sistematização, foi realizada a calagem, para elevar o $\mathrm{pH}$ a 5,5 e semeada pastagem no sistema de cultivo convencional. Para a semeadura foram utilizados 40; 7,5 e $2 \mathrm{~kg} / \mathrm{ha}$ de sementes de L. multiflorum cv. Comum, cornichão (Lotus corniculatus L.) cv. São Gabriel e T. repens cv. Zapican, respectivamente. A adubação, com $\mathrm{P}_{2} \mathrm{O}_{5} \mathrm{e}$ $\mathrm{K}_{2} \mathrm{O}$, em 1997, foi baseada na análise do solo, aplicando-se quantidades de 50, 100 e $150 \%$ da recomendação para $\mathrm{P}_{2} \mathrm{O}_{5}$ e $\mathrm{K}_{2} \mathrm{O}$ (Siqueira et al., 1987), em dois potreiros para cada nível. A dose de adubação correspondente a 100\% foi de 130 e $104 \mathrm{~kg} / \mathrm{ha}$ de $\mathrm{P}_{2} \mathrm{O}_{5}$ e $\mathrm{K}_{2} \mathrm{O}$, respectivamente, sendo aos demais potreiros aplicadas às proporções acima.

Nos anos seguintes (1998 e 1999), realizou-se adubação de manutenção, a lanço, aplicando-se $40 \mathrm{~kg} / \mathrm{ha}$ de $\mathrm{P}_{2} \mathrm{O}_{5}$ e $\mathrm{K}_{2} \mathrm{O}$ nos potreiros com $100 \%$ de recomendação e 150 e $50 \%$ destes nutrientes nos demais potreiros (dois por nível). Nos anos de 1998 e

R. Bras. Zootec., v.34, n.1, p.44-53, 2005 
1999, foram aplicados 101,2 e $90 \mathrm{~kg} / \mathrm{ha}$ de $\mathrm{N}$, respectivamente, em todos os potreiros.

As características químicas do solo, analisadas no ano de 2000, apresentaram os seguintes valores para $\mathrm{pH}\left(\mathrm{H}_{2} \mathrm{O}\right): 6,0,6,2$ e 6,$1 ; \mathrm{K}(\mathrm{mg} / \mathrm{L}): 46,42$ e 42 ; $\mathrm{P}(\mathrm{mg} / \mathrm{L}): 5,5,5,5$ e 13,$0 ; \mathrm{MO}(\%): 2,3,2,0$ e 2,3 , respectivamente para os potreiros que haviam recebido 50, 100 e $150 \%$ da recomendação.

Em abril de 2000, foram aplicados 1,8 L/ha de glifosato para facilitar a emergência das plântulas de L. multiflorum. A adubação de manutenção foi realizada com o objetivo de padronizar os níveis de adubação dos potreiros, sendo aplicados $84 \mathrm{~kg} / \mathrm{ha}$ de $\mathrm{P}_{2} \mathrm{O}_{5}$ nos potreiros com $50 \%$ da recomendação e $14 \mathrm{~kg} / \mathrm{ha}$ de $\mathrm{P}_{2} \mathrm{O}_{5}$ nos demais e $40 \mathrm{~kg} / \mathrm{ha}$ de $\mathrm{K}_{2} \mathrm{O}$ em todos os potreiros. Foi realizada nova semeadura de L. multiflorum no mês de maio, sendo utilizados $25 \mathrm{~kg} / \mathrm{ha}$.

A partir de 2000, após a padronização dos níveis de $\mathrm{K}_{2} \mathrm{O}$ e $\mathrm{P}_{2} \mathrm{O}_{5}$, os tratamentos passaram a ser os seguintes sistemas de manejo: doses de $\mathrm{N}$ combinadas com níveis de suplementação. Os piquetes que correspondiam a 150,100 e $50 \%$ da adubação de $\mathrm{P}_{2} \mathrm{O}_{5}$ e $\mathrm{K}_{2} \mathrm{O}$, receberam 300, 200 e $100 \mathrm{~kg} / \mathrm{ha}$ de $\mathrm{N}$, respectivamente. Os níveis de $\mathrm{N}$ foram subdivididos em cinco aplicações: 01/06; 07/07; 28/07; 02/09 e 07/10. Os níveis de suplementação foram de 0,$8 ; 0,4 \%$ do peso vivo (PV) e sem suplementação. O maior nível foi utilizado nos potreiros com $300 \mathrm{~kg} / \mathrm{ha}$ de N, o nível intermediário para os potreiros com $200 \mathrm{~kg} / \mathrm{ha}$ de N e ausência de suplementação para aqueles de menor nível de N. A suplementação energética fornecida aos animais foi grão de milho moído, visando manter lotação mais elevada nos potreiros com maiores doses de $\mathrm{N}$, já que o sistema de pastejo foi contínuo com lotação fixa. Em virtude de limitações de área e de estrutura para manter animais reguladores, foi utilizada lotação fixa de seis animais por hectare nos tratamentos com 300 e 200 e quatro no tratamento de $100 \mathrm{~kg} /$ ha de N, no período de 15/07 a 03/11/00. Estas lotações basearam-se em uma estimativa da taxa de acúmulo obtida a partir dos anos anteriores (Difante et al., 2001). O peso vivo médio inicial dos novilhos cruza Charolês e Nelore foi de $219 \mathrm{~kg}$. Após este período, houve redução na lotação para dois animais por hectare, em virtude do final do ciclo vegetativo de L. multiflorum e do efeito supressor do herbicida utilizado sobre as espécies nativas, diminuindo de forma drástica sua participação na massa de forragem total disponível.
Para determinação das variáveis morfogênicas e taxa de desfolhanas espécies, foi escolhida aleatoriamente uma área de 0,5 hectare de cada tratamento, onde foram marcadas três transectas paralelas e, em cada uma, cinco quadros de $0,25 \mathrm{~m}^{2}$, eqüidistantemente separados. Nestes quadros, foram marcados três afilhos de cada espécie em estudo. Os afilhos foram marcados com anéis, feitos com fios telefônicos coloridos de um milímetro $(\mathrm{mm}) \mathrm{de}$ espessura. Em virtude das alterações provocadas pelo manuseio das plantas, os quadros e os afilhos foram remarcados a cada quatro avaliações. O intervalo das avaliações foi de sete dias e a troca de afilhos identificados separa os diferentes períodos de avaliação.

No ano de 2000, no início do período de avaliações, havia baixa contribuição de $P$. urvillei nos piquetes, em decorrência da aplicação de glifosato. Em decorrência disso, foram avaliados inicialmente apenas afilhos em L. multiflorum, sendo que as avaliações foram realizadas nos períodos de 19/07 a 09/08, 16/08 a 05/09 e 13/09 a 04/10/00. Com o aumento da contribuição de $P$. urvillei na massa de forragem da área experimental, foram marcados e avaliados afilhos desta espécie nos períodos de 25/10 a 15/11,21/11 a 12/12/00 e 10/01 a 30/01/01. Em cada período foram avaliados 45 afilhos por tratamento, com intervalo das avaliações de sete dias. Ao final de cada período com quatro avaliações, novos afilhos foram remarcados aleatoriamente. A primeira folha de cada afilho foi marcada com corretor ortográfico na sua base, como referencial para as próximas avaliações, assim como folhas que sofreram desfolha (pastejadas) receberam marcação no ápice para poder ser identificada nova desfolha na mesma folha. Em cada avaliação foram verificados os comprimentos de cada folha do afilho, o número de folhas e suas condições, se estavam em alongamento (sem lígula exteriorizada), alongadas (lígula exteriorizada) e se haviam sofrido ou não desfolha.

Para o cálculo da taxa de alongamento foliar (mm/graus-dia/afilho) foi realizado o somatório do alongamento de cada folha no período, e calculada uma média do número de folhas que alongaram no período sem sofrer desfolha. Este valor médio por afilho foi dividido pelo filocrono de cada período.

O filocrono, que representa a soma térmica acumulada em um dado período, para o surgimento de uma folha, foi determinado por regressão entre o número inicial e final de folhas e a soma térmica acumulada de cada período. O filocrono é o valor inverso do coeficiente angular desta regressão, ex-

\section{R. Bras. Zootec., v.34, n.1, p.44-53, 2005}


Tabela 1 - Variáveis meteorológicas registradas durante o experimento (médias dos períodos avaliados) Table 1 - Meteorological variables registered during the trial (average of evaluated periods)

\begin{tabular}{|c|c|c|c|c|c|}
\hline \multirow[t]{2}{*}{$\begin{array}{l}\text { Período } \\
\text { Period }\end{array}$} & \multicolumn{2}{|c|}{$\begin{array}{c}\text { Temperaturas médias do ar }\left({ }^{\circ} \mathrm{C}\right) \\
\text { Average air temperature }\left({ }^{\circ} \mathrm{C}\right)\end{array}$} & \multirow[t]{2}{*}{$\begin{array}{l}\text { Precipitação }(\mathrm{mm}) \\
\text { Precipitation }(\mathrm{mm})\end{array}$} & \multicolumn{2}{|c|}{$\begin{array}{l}\text { Insolação }(\mathrm{h}) \\
\text { Insolation }(h)\end{array}$} \\
\hline & $\begin{array}{l}\text { Máximas } \\
\text { Maximum } \\
\end{array}$ & $\begin{array}{l}\text { Mínimas } \\
\text { Minimum }\end{array}$ & & Total & $\begin{array}{c}\text { Diária } \\
\text { Daily }\end{array}$ \\
\hline \multicolumn{6}{|c|}{ 2000* } \\
\hline $\mathrm{I}$ & 18,1 & 7,0 & 29,6 & 132,9 & 6,0 \\
\hline II & 22,9 & 8,3 & 66,2 & 149,8 & 7,1 \\
\hline III & 22,7 & 12,5 & 240,5 & 109,4 & 5,0 \\
\hline \multicolumn{6}{|c|}{$2000 / 01 * *$} \\
\hline $\mathrm{I}$ & 25,7 & 15,7 & 146,0 & 136,4 & 6,2 \\
\hline II & 29,9 & 17,1 & 128,6 & 177,3 & 8,1 \\
\hline III & 29,2 & 20,7 & 166,0 & 107,0 & 4,9 \\
\hline
\end{tabular}

2000*: I = 19/07 a 09/08; II = 16/08 a 05/09; III = 13/09 a 04/10.

$2000 / 01^{* *}: I=25 / 10$ a $15 / 11$; ||$=21 / 11$ a $12 / 12 / 00 ;$ III = 10/01 a $30 / 01 / 01$.

$2000^{*}: I=07 / 19$ to $08 / 09 ; / I=08 / 16$ to $09 / 05 ; I I I=09 / 13$ to $10 / 04$.

$2000 / 01^{* *}: I=10 / 25$ to $11 / 15$; $I I=11 / 21$ to $12 / 12 / 00$; $I I I=01 / 10$ to $01 / 30 / 01$.

presso em graus-dia (GD). Para o cálculo do filocrono, foi utilizada a temperatura base de $5^{\circ} \mathrm{C}$ para L. multiflorum (Cooper \& Tainton, 1968). Para P. urvillei utilizou-se $8^{\circ} \mathrm{C}$, valor referido por Agnusdei (1999) como a temperatura mínima para o crescimento de folhas em espécies $\mathrm{C}_{4}$ de pastagens naturais.

A taxa de aparecimento de folhas expressa em número de folhas/afilho/dia foi determinada pela divisão entre o número de folhas surgidas no período de avaliação pelo número de dias do período.

A taxa de desfolha foi estimada como média da percentagem do número de folhas pastejadas em cada avaliação no afilho, em relação ao total de folhas do respectivo afilho.

Para avaliar a contribuição de massa de forragem total e das espécies, utilizou-se o método BOTANAL (Tothill et al., 1992), sendo realizadas avaliações mensais, durante todo o período experimental.

O delineamento utilizado foi o inteiramente casualizado, sendo avaliados noventa afilhos por tratamento, quarenta e cinco de cada espécie por repetição. Os afilhos foram testados para a significância da autocorrelação espacial entre eles e, na ausência desta, puderam ser considerados como repetições verdadeiras. Em virtude de os modelos de regressão linear e quadrática não serem significativos, foi utilizada análise de variância multivariada. Para análise da significância dos efeitos de tratamentos e das interações com períodos foram utilizados testes de aleatorização. O método é descrito em Pillar \& Orlóci (1996), implementado com adaptações em rotinas do programa MULTIV (Pillar, 1997), disponível no endereço http://ecoqua.ecologia.ufrgs.br.

\section{Resultados e Discussão}

A massa de forragem de L. multiflorum e sua respectiva contribuição percentual na MS total, na média dos períodos de avaliações, está apresentada na Tabela 2, com o objetivo de caracterizar a estrutura da pastagem ao longo das avaliações.

No período de julho a outubro, a contribuição percentual de L. multiflorum na massa de forragem, na média dos tratamentos, nas datas iniciais de avaliação e no último levantamento, foram de 84,4; 90,3; 88,8 e $66,6 \%$, respectivamente. Em 1999, na mesma área, esta contribuição, na média dos tratamentos, variou de 3,3 a $11,4 \%$, para o período de junho a novembro (Pigatto, 2001). O aumento na contribuição percentual na massa de forragem do ano de 2000 foi influenciado principalmente pela aplicação de herbicida na área, o que reduziu a participação das demais espécies presentes na área (nativas e/ou introduzidas), as quais possuíam elevada contribuição na MS total no ano de 1999 (Pigatto, 2001), entre elas P. urvillei, Ciperáceas e T. repens.

O uso da suplementação energética teve como objetivo produzir efeito substitutivo no consumo de forragem para permitir aumento da carga animal. As menores taxas de desfolha no tratamento com os maiores níveis de $\mathrm{N}$ e suplemento, no início das avaliações (19/07 a 09/08), são indicativos deste efeito substitutivo (Tabela 3). Neste tratamento, o aumento da participação de L. multiflorum no segundo período (Tabela 2) e a maior carga animal observada no último período (Difante et al., 2001), adotando-se lotação fixa, devem ter contribuído

R. Bras. Zootec., v.34, n.1, p.44-53, 2005 
Tabela 2 - Massa de forragem média (kg/ha de MS) de Lolium multiflorum e contribuição percentual na MS total (\%), nos diferentes períodos de avaliação, em área de várzea, submetidos a níveis de $\mathrm{N}$

Table 2 - Average forage mass ( $\mathrm{kg} / \mathrm{ha}$ of DM) of Lolium multiflorum and percent contribution in the DM total (\%), in the different periods of evaluation, in lowland area, submitted to levels of $\mathrm{N}$

\begin{tabular}{|c|c|c|c|c|c|c|}
\hline \multirow[t]{3}{*}{ N (kg/ha) } & \multicolumn{6}{|c|}{$\begin{array}{c}\text { Matéria seca }(\mathrm{kg} / \mathrm{ha}) \\
\text { Dry matter }(\mathrm{kg} / \mathrm{ha})\end{array}$} \\
\hline & $13 / 07-15 / 08$ & $\%$ & $15 / 08-12 / 09$ & $\%$ & 12/09-24/10 & $\%$ \\
\hline & $07 / 13-08 / 15$ & & $08 / 15-09 / 12$ & & $09 / 12-10 / 24$ & \\
\hline 100 & 784 & 79 & 697 & 81 & 1087 & 76 \\
\hline 200 & 1004 & 95 & 814 & 92 & 944 & 80 \\
\hline 300 & 1033 & 89 & 1092 & 96 & 1101 & 77 \\
\hline
\end{tabular}

Tabela 3 - Taxa de desfolha (\% folhas desfolhadas/afilho) em Lolium multiflorum Lam. em área de várzea, submetidos a níveis de $\mathrm{N}$ combinadas com níveis de suplementação

Table 3 - Defoliation rates (\% leaves defoliated/tiller) in Lolium multiflorum Lam. in a lowland area, submitted to $\mathrm{N}$ levels combined with supplementation levels

\begin{tabular}{|c|c|c|c|}
\hline \multirow[t]{3}{*}{$\begin{array}{l}\text { Níveis de } \mathrm{N} \text { e suplementação } \\
\text { Levels of } N \text { and supplementation }\end{array}$} & \multicolumn{3}{|c|}{$\begin{array}{c}\text { Taxa de desfolha (\% folhas desfolhadas/afilho) } \\
\text { Defoliation rate (\% leaves defoliated/tiller) }\end{array}$} \\
\hline & 19/07 a 09/08 & $16 / 08$ a 05/09 & $13 / 09$ a 04/10/00 \\
\hline & $07 / 19$ to $08 / 09$ & $08 / 16$ to $09 / 05$ & $09 / 13$ to $10 / 04 / 00$ \\
\hline $100+0,0 *$ & $25,31 b^{1}$ & $29,73 \mathrm{~b}$ & $31,54 \mathrm{~b}$ \\
\hline $200+0,4$ & $30,22 \mathrm{a}$ & $35,90 a$ & $36,16 \mathrm{a}$ \\
\hline $300+0,8$ & $18,31 \mathrm{c}$ & $29,59 b$ & $36,01 \mathrm{a}$ \\
\hline Média (Average) & 24,61 & 31,74 & 34,57 \\
\hline $\mathrm{CV}(\%)$ & 41,78 & 3,13 & 27,52 \\
\hline
\end{tabular}

${ }^{1}$ Letras minúsculas distintas na mesma coluna diferem entre si $(P=0,054)$, pelo teste de aleatorização.

1 Different small letters in the same column differ among them $(P=.054)$, by randomization test.

* $100 \mathrm{~kg} / \mathrm{ha}$ de N + 0,0\% PV (100 kg/ha of $+.0 \%$ LW); $200 \mathrm{~kg} / \mathrm{ha}$ de N + 0,4\% PV $(200 \mathrm{~kg} / \mathrm{ha}$ of $\mathrm{N}+.4 \% \mathrm{LW}) ; 300 \mathrm{~kg} / \mathrm{ha} \mathrm{de} \mathrm{N} \mathrm{+} \mathrm{0,8 \%} \mathrm{PV}$ $(300 \mathrm{~kg} / \mathrm{ha}$ of $\mathrm{N}+.8 \% \mathrm{LW})$.

para redução deste efeito substitutivo e aumento nas taxas de desfolha.

A taxa de desfolha observada no tratamento intermediário $(200 \mathrm{~kg} / \mathrm{ha}$ de $\mathrm{N}+0,4 \% \mathrm{PV}$ de suplemento) foi superior aos demais nos meses de julho a setembro. Os resultados observados para este tratamento sugerem que pode ter ocorrido efeito aditivo do suplemento sobre o consumo de forragem, em razão de o nível de suplementação utilizada ter sido menor (Rocha, 1998).

Os dados de desempenho animal, consumo e produção de massa de forragem não são discutidos no presente trabalho, pois não foram o foco da pesquisa. Os mesmos podem ser observados em Difante et al. (2001).

$\mathrm{Na}$ taxa de alongamento de L. multiflorum (Tabela 4), houve diferença entre tratamentos nos dois primeiros períodos de avaliação, observando-se os maiores valores com o uso de $300 \mathrm{~kg} / \mathrm{ha}$ de N. A semelhança entre os níveis de 100 e $200 \mathrm{~kg} / \mathrm{ha}$ de $\mathrm{N}$ pode estar relacionada com a adubação utilizada, visando tornar semelhantes os níveis de $\mathrm{P}_{2} \mathrm{O}_{5}$. $\mathrm{O}$ tratamento com menor nível de $\mathrm{N}$ recebeu cerca de seis vezes mais $\mathrm{P}_{2} \mathrm{O}_{5}$ que os demais tratamentos. Duru et al. (2000) observaram relação da resposta à adubação nitrogenada e fosfatada, em que o nível ótimo de um desses elementos potencializa a utilização do outro. O efeito do fósforo ocorre com o aumento da mineralização da matéria orgânica, que eleva a disponibilidade de $\mathrm{N}$ na solução do solo.

$\mathrm{O}$ terceiro período apresentou valores próximos entre os tratamentos, em razão deste período coincidir com a fase de alongamento dos entrenós, quando a prioridade de alocação de fotoassimilados passa a ser o crescimento das estruturas reprodutivas e as lâminas foliares reduzem sua taxa de expansão (Skinner \& Nelson, 1995).

Entre os períodos, as taxas de alongamento foram superiores no primeiro em relação aos demais, e do segundo em relação ao terceiro. Estas diferenças podem ser justificadas pelo estádio fenológico da 
Tabela 4 - Taxa de alongamento (mm de lâmina foliar/GD/afilho), taxa de aparecimento de lâminas foliares (folhas/ afilho/dia), taxa de desfolha (\% de folhas pastejadas/afilho) e intervalo médio para surgimento de folhas (dias) de Lolium multiflorum Lam. em área de várzea, submetidos a níveis de $\mathrm{N}$

Table 4 - Leaf elongation rates ( $\mathrm{mm}$ of leaf lamina/DD/tiller), leaf laminas appearance rates (leaf number/tiller/day), defoliation rates (\% of leaves grazed/tiller) and average leaf appearance interval (days) of Lolium multiflorum Lam. in lowland area, submitted to levels of $N$

\begin{tabular}{|c|c|c|c|}
\hline \multirow[t]{3}{*}{$\mathrm{N}(\mathrm{kg} / \mathrm{ha})$} & \multicolumn{3}{|c|}{$\begin{array}{l}\text { Taxa de alongamento ( } \mathrm{mm} \text { de lâmina foliar/GD/afilho) } \\
\text { Elongation rate (mm of leaf lamina/DD/tiller) }\end{array}$} \\
\hline & 19/07 a 09/08 & 16/08 a 05/09 & $13 / 09$ a 04/10/00 \\
\hline & $07 / 19$ to $08 / 09$ & $08 / 16$ to $09 / 05$ & $09 / 13$ to $10 / 04 / 00$ \\
\hline 100 & $0,564 b^{1}$ & $0,388 \mathrm{~b}$ & 0,229 \\
\hline 200 & $0,524 b$ & $0,368 b$ & 0,247 \\
\hline 300 & $0,617 \mathrm{a}$ & $0,497 \mathrm{a}$ & 0,203 \\
\hline Média (Average) & $0,568 \mathrm{~A}^{2}$ & $0,417 \mathrm{~B}$ & $0,226 \mathrm{C}$ \\
\hline $\mathrm{CV}(\%)$ & 44,40 & 54,07 & 92,25 \\
\hline
\end{tabular}

\begin{tabular}{|c|c|c|c|}
\hline & \multicolumn{3}{|c|}{$\begin{array}{c}\text { Taxa de aparecimento ( } \mathrm{n}-\mathrm{o} \text { de folhas/afilho/dia) } \\
\text { Appearance rate(leaf number/tiller/day) }\end{array}$} \\
\hline 100 & $0,080 \mathrm{a}^{1}$ & 0,077 & $0,062 b$ \\
\hline 200 & $0,075 \mathrm{ab}$ & 0,075 & $0,071 \mathrm{a}$ \\
\hline 300 & $0,072 b$ & 0,078 & $0,055 b$ \\
\hline Média (Average) & $0,076 A^{2}$ & $0,077 \mathrm{~A}$ & $0,063 \mathrm{~B}$ \\
\hline $\mathrm{CV}(\%)$ & 52,26 & 51,09 & 55,74 \\
\hline
\end{tabular}

Intervalo médio para surgimento de folhas (dias)

Average interval for leaf appearence (days)

\begin{tabular}{lccc}
\hline 100 & $12,5 \mathrm{a}$ & 13,0 & 16,1 \\
200 & $13,3 \mathrm{ab}$ & 13,3 & 14,1 \\
300 & $13,9 \mathrm{~b}$ & 12,8 & 18,2 \\
Média (Average) & $13,1 \mathrm{~A}$ & $13,0 \mathrm{~A}$ & $15,8 \mathrm{~B}$ \\
CV $(\%)$ & 52,26 & 51,09 & 55,74 \\
\hline
\end{tabular}

${ }_{1}^{1}$ Letras minúsculas distintas na mesma coluna diferem entre si $(P<0,095)$ pelo teste de aleatorização.

${ }^{2}$ Letras maiúsculas distintas na mesma linha diferem entre si $(P<0,003)$ pelo teste de aleatorização.

${ }^{1}$ Different small letters in the same column differ among them $(P<.095)$ by randomization test.

2 Different capital letters in the same line differ among them $(P<.003)$ by randomization test.

planta, em que a prioridade das folhas para utilização de fotossintatos e nutrientes é gradativamente reduzida. Além disso, o período inicial coincide com o maior volume $(60 \%$ do total) de $\mathrm{N}$ aplicado. As taxas observadas neste trabalho são superiores às relatadas, na mesma área, por Pigatto (2001), no ano anterior, onde foram avaliados níveis de adubação de $\mathrm{P}_{2} \mathrm{O}_{5}$ e $\mathrm{K}_{2} \mathrm{O}$ com adubação nitrogenada fixa de $90 \mathrm{~kg} / \mathrm{ha}$. Os valores médios dos tratamentos observados naqueles períodos foram de 0,$452 ; 0,410 \mathrm{e}$ $0,232 \mathrm{~mm}$ de lâmina foliar/GD/afilho, respectivamente para 29/06 a 20/07, 03/08 a 17/08 e 14/09 a 05/10/99. A maior diferença nas taxas de alongamento foi observada no período inicial de crescimento, no qual havia sido aplicado cerca de três vezes mais $\mathrm{N}$, na média dos tratamentos de 2000, em relação aos de 1999 (120 x 45 kg/ha). Nos demais períodos, não foram observadas diferenças entre os valores médios dos tratamentos, apesar de haver níveis de $\mathrm{N}$ duas vezes maiores, na média de 2000, em relação a 1999. Este fato demonstra a importância da adubação nitrogenada ser aplicada no estádio inicial de desenvolvimento desta espécie (Gonçalves \& Quadros, 2003).

Os valores observados para taxa de alongamento foliar no primeiro período são semelhantes aos relatados por Bandinelli et al. (2002), que verificaram para L. multiflorum valores de 0,554 e $0,546 \mathrm{~mm} /$ GD/afilho para os tratamentos de 100 e $200 \mathrm{~kg} / \mathrm{ha}$ de $\mathrm{N}$, no período de $03 / 08$ a 21/08/01, no qual já haviam aplicado 66,7 e 133,4 kg/ha de $\mathrm{N}$ até a última data (21/08). Esses autores observaram maior redução nesta taxa a partir do período de 28/08 a 19/09/01, confirmando a sugestão de antecipar a aplicação de N (Gonçalves \& Quadros, 2003).

Para a taxa de aparecimento de folhas houve diferença entre os tratamentos no primeiro e terceiro períodos avaliados (Tabela 4). No primeiro, o tratamento $100 \mathrm{~kg} / \mathrm{ha}$ de $\mathrm{N}$ apresentou a maior taxa de aparecimento, sendo necessários em média 12,5 dias para o aparecimento de nova folha. O tratamento $300 \mathrm{~kg} / \mathrm{ha}$ de $\mathrm{N}$ apresentou a menor taxa, sendo

R. Bras. Zootec., v.34, n.1, p.44-53, 2005 
necessários, em média, 13,9 dias para o aparecimento de nova folha. Comparando-se as taxas de alongamento e aparecimento de folhas neste primeiro período, para o nível de $300 \mathrm{~kg} / \mathrm{ha}$ de $\mathrm{N}$, constata-se a relação inversa existente entre essas taxas, também observadas por Zarrough et al., 1984; Pigatto, 2001; Gonçalves \& Quadros, 2003.

Alguns autores não observaram esta relação, Lattanzi et al. (1996), estudando o efeito da fertilização nitrogenada sobre a morfogênese de L. multiflorum, observaram que a fertilização nitrogenada propiciou incremento na velocidade de aparecimento de folhas em $30 \%$. Esta resposta foi significativa até a dose de $150 \mathrm{~kg} /$ ha de $\mathrm{N}$, que correspondeu ao conteúdo de $\mathrm{N}$ em que a planta duplicou o da testemunha.

No segundo período, as taxas de aparecimento foram semelhantes. No terceiro, o tratamento com $200 \mathrm{~kg} / \mathrm{ha}$ de $\mathrm{N}$ apresentou a maior taxa de aparecimento de folhas, sendo necessários, em média, 14,1 dias para o aparecimento de nova folha. Os tratamentos com 100 e $300 \mathrm{~kg} / \mathrm{ha}$ de $\mathrm{N}$ apresentaram as menores taxas, sendo necessários em média $16,1 \mathrm{e}$ 18,2 dias para o aparecimento de nova folha. O tratamento com $200 \mathrm{~kg} / \mathrm{ha}$ de $\mathrm{N}$ manteve o intervalo do surgimento de novas folhas mais constante, graças à uniformidade da taxa de desfolha (Tabela 3).

Os intervalos de surgimento de folhas (Tabela 4) são semelhantes aos observados por Gonçalves \& Quadros (2003), que relataram valores entre 12,7 a 15,6 dias, trabalhando com doses de $\mathrm{N}$ de 90,120 e $180 \mathrm{~kg} / \mathrm{ha}$.

Entre os períodos, houve diferença $(\mathrm{P}=0,0001)$ do terceiro período em relação aos demais, tendo este apresentado, em média, a menor taxa de aparecimento de folhas, 15,8 dias, o que está relacionado com a aproximação do final do ciclo vegetativo. Com o avanço dos estádios de desenvolvimento, a planta realoca as prioridades na distribuição de fotossintatos e nutrientes para os meristemas florais, o que tende a reduzir a formação de novas folhas (Skinner \& Nelson, 1995).

Os valores médios de filocrono para $L$. multiflorum, nos três períodos avaliados, são apresentados na Tabela 5. O terceiro período apresentou o maior valor de filocrono, seguido do segundo e primeiro períodos, respectivamente. $\mathrm{O}$ aumento progressivo de filocrono é consequiência das maiores temperaturas médias observadas, o que amplia a soma térmica com o avanço do ciclo da pastagem.

No terceiro período, o maior valor de filocrono observado também é decorrência de maior necessidade de acúmulo de temperatura para o alongamento dos entrenós.

Lattanzi et al. (1996) verificaram valores de filocrono de $120 \mathrm{GD} /$ folha para L. multiflorum, com $100 \mathrm{~kg} / \mathrm{ha}$ de $\mathrm{N}$ e $110 \mathrm{GD} /$ folha quando utilizados $200 \mathrm{~kg} / \mathrm{ha}$ de $\mathrm{N}$. Esses autores observaram que a resposta foi significativa até o nível de $150 \mathrm{~kg} /$ ha de N.

Os valores de filocrono do primeiro e segundo períodos, independentemente de tratamento, são semelhantes aos observados por Viégas (1998). Bandinelli et al. (2002), no período de 03/08 a 21/08 e 28/08 a 19/09, encontraram valores de filocrono variando de 144,7 a 149,2 e 136,8 a $152,0 \mathrm{GD}$, respectivamente, usando 100 e $200 \mathrm{~kg} / \mathrm{ha}$ de $\mathrm{N}$.

Na Tabela 6, pode ser observada a massa média de forragem e a contribuição percentual de $P$. urvillei, nos respectivos períodos de avaliação. Em virtude da aplicação do herbicida glifosato, $P$. urvillei possuía no final de outubro valores de 0,$21 ; 1,42$ e $0,01 \%$ de contribuição na MS total disponível na área (média de $1644 \mathrm{~kg} / \mathrm{ha}$ de MS), respectivamente, para os tratamentos com 100, 200 e $300 \mathrm{~kg} / \mathrm{ha}$ de $\mathrm{N}$ (Tabela 6).

Tabela 5 - Filocrono (GD) de Lolium multiflorum Lam. em área de várzea, submetidos a níveis de $\mathrm{N}$ Table 5 - Phyllochron (DD) of Lolium multiflorum Lam. in lowland area, submitted the levels of $N$

\begin{tabular}{|c|c|c|c|c|}
\hline \multirow[t]{2}{*}{$\mathrm{N}(\mathrm{kg} / \mathrm{ha})$} & \multicolumn{3}{|c|}{$\begin{array}{l}\text { Filocrono (GD) } \\
\text { Phyllochron }(D D)\end{array}$} & \multirow[t]{2}{*}{$\begin{array}{l}\text { Média/tratamento } \\
\text { Treatment average }\end{array}$} \\
\hline & $\begin{array}{l}19 / 07 \text { a } 09 / 08 \\
07 / 19 \text { to } 08 / 09 \\
\end{array}$ & $\begin{array}{l}16 / 08 \text { a } 05 / 09 \\
08 / 16 \text { to } 09 / 05\end{array}$ & $\begin{array}{l}13 / 09 \text { a } 04 / 10 / 00 \\
09 / 13 \text { to } 10 / 04 / 00\end{array}$ & \\
\hline 100 & 96,9 & 133,0 & 197,0 & 142,3 \\
\hline 200 & 98,9 & 156,2 & 177,8 & 144,3 \\
\hline 300 & 99,9 & 131,2 & 261,5 & 164,2 \\
\hline Média/ período & $98,6 \mathrm{C}^{1}$ & $140,1 \mathrm{~B}$ & $212,1 \mathrm{~A}$ & \\
\hline \multicolumn{5}{|l|}{ Period average } \\
\hline $\mathrm{CV}(\%)$ & 52,26 & 51,09 & 55,74 & 53,12 \\
\hline
\end{tabular}

\footnotetext{
${ }^{1}$ Letras maiúsculas distintas na mesma linha diferem entre si $(P<0,014)$ pelo teste de aleatorização.

1 Different capital letters in the same line differ $(P<.014)$ by randomization test.
}

\section{R. Bras. Zootec., v.34, n.1, p.44-53, 2005}


Este período apresentou máxima contribuição de L. multiflorum (Tabela 2), mas foi observada a maior taxa de desfolha em P. urvillei (Tabela 7), demonstrando a elevada preferência pela espécie por parte dos animais, conforme observado em trabalho anterior (Pigatto, 2001).

No final de novembro, essa contribuição passou para 8,$20 ; 11,22$ e $1,21 \%$, respectivamente para os tratamentos 100,200 e $300 \mathrm{~kg} /$ ha de N, sem contribuição significativa de L. multiflorum, correspondendo a $1.854 \mathrm{~kg} / \mathrm{ha}$ de MS a massa de forragem média nos tratamentos. Nesse período, houve redução na taxa de desfolha, influenciada principalmente pelo fato de, a partir de $03 / 11$, ter sido reduzida a carga animal na área (dois animais/ha). Outro fator que influenciou esta variável foi o aumento na contribuição de outras espécies nativas na área, entre elas Echinochloa spp., Leersia hexandra Sw. e Panicum milioides Nees ex. Trin., e a participação de T. repens L., espécie introduzida.
Em dezembro, a contribuição passou para 18,57; 16,52 e 5,85\% na MS total, na ordem crescente dos tratamentos, sendo a massa de forragem média dos tratamentos de $2.528 \mathrm{~kg} / \mathrm{ha}$ de MS. No último período (janeiro), a contribuição na MS total de $P$. urvillei foi de: 17,$18 ; 19,50$ e $7,19 \%$, respectivamente, nos tratamentos com 100, 200 e $300 \mathrm{~kg} / \mathrm{ha}$ de $\mathrm{N}$, sendo que a massa de forragem média dos tratamentos foi de $2.679 \mathrm{~kg} / \mathrm{ha}$ de MS. Nos dois últimos períodos, houve estabilização tanto na contribuição percentual de P. urvillei quanto na taxa de desfolha, essa última, principalmente em função da manutenção da mesma carga animal entre os tratamentos.

A taxa de alongamento foliar do tratamento com $300 \mathrm{~kg} / \mathrm{ha}$ de $\mathrm{N}$ foi superior ao menor nível de $\mathrm{N}$ $(\mathrm{P}<0,065)$, em todos os períodos (Tabela 8), e não diferiu do nível intermediário apenas no segundo período. Isso demonstra a resposta da taxa de alongamento de lâminas foliares de $P$. urvillei ao uso de $\mathrm{N}$, apesar da ausência de significância para os modelos lineares e quadráticos. Entretanto, estas taxas

Tabela 6 - Massa de forragem média (kg/ha MS) mensal de Paspalum urvillei e contribuição percentual na MS total (\%), nos meses de outubro (24/10), novembro (28/11), dezembro (28/12/01) e janeiro (30/01/01), em área de várzea, submetidos a níveis de N (100, 200 e $300 \mathrm{~kg} / \mathrm{ha})$

Table 6 - Average forage mass ( $\mathrm{kg} / \mathrm{ha} \mathrm{DM}$ ) of Paspalum urvillei and percent contribution in the total DM (\%), in October (10/24), November (11/28), December (12/28/01) and January (01/30/01) in lowland area, submitted to $N$ levels $(100,200$ and $300 \mathrm{~kg} / \mathrm{ha})$

\begin{tabular}{lrrrrrrrr}
\hline $\mathrm{N}(\mathrm{kg} / \mathrm{ha})$ & \multicolumn{8}{c}{$\begin{array}{c}\text { Matéria seca }(\mathrm{kg} / \mathrm{ha}) \\
\text { Dry matter }(\mathrm{kg} / \mathrm{ha})\end{array}$} \\
\cline { 2 - 10 } & Out & $\%$ & Nov & $\%$ & Dez & $\%$ & Jan & $\%$ \\
& Oct & & Nov & & Dec & & Jan & \\
\hline 100 & 4 & 0,21 & 171 & 8,20 & 298 & 18,57 & 523 & 17,18 \\
200 & 21 & 1,42 & 167 & 11,22 & 220 & 16,52 & 404 & 19,50 \\
300 & 1 & 0,01 & 24 & 1,21 & 100 & 5,85 & 210 & 7,19 \\
\hline
\end{tabular}

Tabela 7 - Taxa de desfolha (\% folhas desfolhadas/afilho) em Paspalum urvillei em área de várzea, submetidos a doses de $\mathrm{N}$ combinadas com níveis de suplementação

Table 7 - Defoliation rates (\% leaves defoliated/tiller) in Paspalum urvillei in a lowland area, submitted to $\mathrm{N}$ levels combined with supplementation levels

\begin{tabular}{cccc}
\hline $\begin{array}{l}\text { Níveis de N e suplementação } \\
\text { Levels of } N \text { and supplementation }\end{array}$ & \multicolumn{3}{c}{$\begin{array}{c}\text { Taxa de desfolha (\% folhas desfolhadas/afilho) } \\
\text { Defoliation rate (\% leaves defoliated/tiller) }\end{array}$} \\
\cline { 2 - 4 } & $25 / 10$ a 15/11 & $21 / 11$ a 12/12/00 & $10 / 01$ a $30 / 01 / 01$ \\
& $10 / 25$ to $11 / 15$ & $11 / 21$ to $12 / 12 / 00$ & $01 / 10$ to $01 / 30 / 01$ \\
\hline $100+0,0 *$ & 44,5 & 32,6 & 38,3 \\
$200+0,4$ & 40,2 & 30,6 & 34,9 \\
$300+0,8$ & 41,6 & 31,3 & 35,8 \\
CV $(\%)$ & 38,6 & 21,5 & 34,1
\end{tabular}

\footnotetext{
* $100 \mathrm{~kg} / \mathrm{ha}$ de $\mathrm{N}+0,0 \% \mathrm{PV}(100 \mathrm{~kg} / \mathrm{ha}$ of $\mathrm{N}+.0 \% \mathrm{LW})$.

$200 \mathrm{~kg} / \mathrm{ha}$ de $\mathrm{N}+0,4 \% \mathrm{PV}(200 \mathrm{~kg} / \mathrm{ha}$ of $\mathrm{N}+.4 \% \mathrm{LW})$.

$300 \mathrm{~kg} / \mathrm{ha}$ de $\mathrm{N}+0,8 \% \mathrm{PV}(300 \mathrm{~kg} / \mathrm{ha}$ of $\mathrm{N}+.8 \% \mathrm{LW})$.
}

R. Bras. Zootec., v.34, n.1, p.44-53, 2005 
Tabela 8 - Taxas de alongamento de folhas ( $\mathrm{mm}$ de lâmina foliar/GD/afilho) e filocrono (GD) médio de folhas de Paspalum urvillei Steud., em área de várzea, submetidos a níveis de $\mathrm{N}$

Table 8 - Leaf elongation rates ( $\mathrm{mm}$ of leaf lamina/DD/tiller) and average phyllochron (DD) of leaves of Paspalum urvillei Steud. in lowland area, submitted to levels of $N$

\begin{tabular}{|c|c|c|c|c|}
\hline \multirow[t]{3}{*}{$\mathrm{N}(\mathrm{kg} / \mathrm{ha})$} & \multicolumn{3}{|c|}{$\begin{array}{l}\text { Taxa de alongamento ( } \mathrm{mm} \text { de lâmina foliar/GD/afilho) } \\
\text { Leaf elongation rates ( } \mathrm{mm} \text { of leaf lamina/DD/tiller) }\end{array}$} & \multirow{3}{*}{$\begin{array}{c}\text { Filocrono (GD) } \\
\text { Phyllochron }(D D)\end{array}$} \\
\hline & $25 / 10$ a $15 / 11$ & $21 / 11$ a $12 / 12 / 00$ & $10 / 01$ a $30 / 01 / 01$ & \\
\hline & $10 / 25$ to $11 / 15$ & $11 / 21$ to $12 / 12 / 00$ & $01 / 10$ to $01 / 30 / 01$ & \\
\hline 100 & $0,212 b^{1}$ & $0,214 b$ & $0,260 \mathrm{~b}$ & 196,8 \\
\hline 200 & $0,221 b$ & $0,264 a$ & $0,244 b$ & 202,3 \\
\hline 300 & $0,273 \mathrm{a}$ & $0,248 \mathrm{a}$ & $0,341 \mathrm{a}$ & 207,2 \\
\hline $\mathrm{CV}(\%)$ & 42,5 & 54,2 & 28,9 & 61,5 \\
\hline
\end{tabular}

${ }^{1}$ Letras minúsculas distintas na mesma coluna diferem entre si $(P<0,065)$ pelo teste de aleatorização.

${ }^{1}$ Different small letters in the same column differ among them $(P<.065)$ by randomization test.

foram inferiores àquelas observadas por Pigatto (2001), com aplicação de $90 \mathrm{~kg} / \mathrm{ha}$ de N. Em virtude de as plantas de $P$. urvillei apresentarem menor número de afilhos (três a quatro), em comparação às touceiras do ano anterior, parte do $\mathrm{N}$ pode ter sido translocada para raízes e rizomas em formação, o que pode ter diminuído a resposta na taxa de alongamento foliar. As touceiras desenvolvidas com cerca de 10 a 15 afilhos do ano de 1999 foram suprimidas pela aplicação de glifosato, o que alterou sua resposta morfogênica, no segundo ano.

O filocrono médio dos tratamentos calculado por período (Tabela 8) variou de 196,8 a 207,2 GD, valores intermediários aos observados por Pigatto (2001), que variaram de 166 a $250 \mathrm{GD}$, o que demonstra que esta variável não sofreu o efeito da adubação a base de NPK.

A taxa média de aparecimento de folhas de P. urvillei foi de 0,07 folhas/dia, não apresentando diferenças entre os tratamentos. O intervalo médio (tratamentos e períodos) do aparecimento de nova folha foi de 14,1 dias, sendo influenciada principalmente pela soma térmica acumulada. Os valores de taxa de aparecimento foram inferiores aos observados por Pigatto (2001), que foram na média de períodos e tratamentos de 0,074 folhas/dia e intervalo médio de 13,5 dias para o surgimento de nova folha.

\section{Conclusões}

Para aumentar as taxas de alongamento de Lolium multiflorum, deve ser recomendada a aplicação de maiores doses da adubação nitrogenada nos estádios iniciais de desenvolvimento desta espécie.
O nível de adubação de $300 \mathrm{~kg} / \mathrm{ha}$ de $\mathrm{N}$ mantém altas taxas de alongamento foliar em touceiras de Paspalum urvillei em formação, as quais apresentam apenas afilhos em desenvolvimento.

A taxa de desfolha de Paspalum urvillei é elevada, independentemente de sua contribuição total na massa de forragem disponível.

A adubação nitrogenada não interfere no filocrono de ambas as espécies.

O manejo a ser adotado em áreas de várzea deve considerar o conhecimento das espécies de alto potencial de produção no sistema, principalmente em função da disponibilidade de nutrientes.

\section{Literatura Citada}

AGNusDeI, M. Analyse de la dynamique de la morphogenèse foliaire et de la défoliation de plusieurs espèces de graminées soumises à un pâturage continu dans une communauté végétale de la Pampa Humide (Argentine). Nancy: Institut National Polytechnique de Lorraine, 1999. 108p. Thèse (Doctorat-Agronomie) - Institut National Polytechnique de Lorraine, 1999.

BANDINELLI, D.G.; QUADROS, F.L.F.; ROCHA, M.G. et al. Variáveis morfogênicas de aveia (Avena strigosa) e azevém (Lolium multiflorum), sob pastejo e níveis de adubação nitrogenada. In: REUNIÃO ANUAL DA SOCIEDADE BRASILEIRA DEZOOTECNIA, 39., 2002, Recife. Anais... Recife: TechnoMedia, 2002. CD-ROM. Forragicultura. [02sbz1012.pdf].

BANDINELli, D.G.; QUADROS, F.L.F.; PIGATTO, A.G.S. et al. Avaliação da contribuição de espécies forrageiras cultivadas e nativas na dinâmica do terceiro ano da vegetação de várzea sob três níveis de adubação. In: REUNIÃO ANUAL DA SOCIEDADE BRASILEIRA DE ZOOTECNIA, 37., 2000, Viçosa, MG. Anais... Viçosa, MG: Gnosis, 2000. CD-ROM. Forragicultura. [For 2 -072]. 
COOPER, J.P.; TAINTON, N.M. Light and temperature requirements for the growth of tropical and temperate grasses. Herbage Abstracts, v.38, p.167-176, 1968.

CORSI, M.; NASCIMENTO JR., D. Princípios de fisiologia e morfologia de plantas forrageiras aplicados ao manejo das pastagens. In: PEIXOTO, A.M.; MOURA, J.C.; FARIA, V.R. (Eds.) Pastagens: fundamentos da exploração racional. Piracicaba: Fundação de Estudos Agrários Luiz de Queiroz, 1994. p.15-48.

CRUZ, P.; BOVAL, M. Effect of nitrogen on some morphogenetical traits of temperate and tropical perennial forage grasses. In: SIMPÓSIO INTERNACIONAL GRASSLAND ECOPHYSIOLOGY AND GRAZING ECOLOGY, 1999, Curitiba. Anais... Curitiba: Universidade Federal do Paraná, 1999. p.134-150.

DIFANTE, G.S.; MARCHEZAN, E.; MARZARI, V. et al. Produção de novilhos de corte suplementados em pastagem de inverno, submetida a doses de adubação nitrogenada em área de várzea. In: REUNIÃO ANUAL DA SOCIEDADE BRASILEIRA DE ZOOTECNIA, 38., 2001, Piracicaba. Anais... Piracicaba: Fundação de Estudos Agrários Luiz de Queiroz, 2001. p.375-376.

DURU, M.; MAGDA, D.; CRUZ, P. et al. Relation entre la composition botanique et la valeur d'usage d'une prairie. Application pour la définition d'itinéraires techniques adaptes a differents objectifs. Exemple des prairies permanentes fauchees et paturees dans les pyrenees centrales. In: REUNIÃO DO GRUPO TÉCNICO EM FORRAGEIRAS DO CONE SUL - ZONA CAMPOS, 18., 2000, Guarapuava. Anais... Guarapuava: CPAF/FAPA, 2000. p.44-60.

EMPRESA BRASILEIRA DE ESTUDOS AGROPECUÁRIOS - EMBRAPA. Centro Nacional de Pesquisa de Solos. Sistema brasileiro de classificação de solos. Brasília: Embrapa, 1999. 412p.

GILLEN, R.L.; McCOLLUM, F.T.; BRUMMER, J.E. Tiller defoliation patterns under short duration grazing in tallgrass prairie. Journal of Range Management, v.43, n.2, p.95-99, 1990.

GONÇALVES, E.N.; QUADROS, F.L.F. Características morfogênicas de azevém anual (Lolium multiflorum Lam.) sob pastejo em sistemas intensivos de utilização. Ciência Rural, v.33, n.6, p.1129-1134, 2003.

LATTANZI, F.; MARINO, M.A.; MAZZANTI, A. Efecto de la fertilizacion nitrogenada sobre la morfogenesis de raigras anual cv. Grasslands Tama. Revista Argentina de Producción Animal, v.16, n.1, p.240-241, 1996.

LEMAIRE, G.; CHAPMAN, D. Tissue flows in grazed plant communities. In: HODGSON, J; ILLIUS, A.W. (Eds.) The ecology and management of grazing systems. Wallingford: CAB International, 1996. p.3-36.

MARCHEZAN, E.; VIZZOTTO, V.R.; ZIMMERMAN, F.L. Produção de forrageiras de inverno em diferentes espaçamentos entre drenos superficiais sob pastejo animal em várzea. Ciência Rural, v.28, n.3, p.393-397, 1998.
MAZZANTI, A. Adaptacion de especies forrajeras a la defoliacion. In: SIMPÓSIO SOBRE AVALIAÇÃO DE PASTAGENS COM ANIMAIS, 1., 1997, Maringá. Anais... Maringá: Cooper Graf. Artes Gráficas Ltda., 1997. p.5-84.

NABINGER, C. Pastagens cultivadas como alternativas para áreas de várzea. In: SIMPÓSIO SOBRE ALTERNATIVA AO SISTEMA TRADICIONAL DE UTILIZAÇÃO DE VÁRZEAS DO RIO GRANDE DO SUL, 1., 1984, Porto Alegre. Anais... Brasília: PROVÁRZEAS/PROFIR, 1986. p.220-232.

PIGATTO, A.G.S. Dinâmica da vegetação e do crescimento de Lolium multiflorum Lam. (azevém anual) e Paspalum urvillei Steud., em área de várzea, sob pastejo por bovinos. Santa Maria: Universidade Federal de Santa Maria, 2001. 101p. Dissertação (Mestrado em Zootecnia) - Universidade Federal de Santa Maria, 2001.

PILLAR, V.D.P. Multivariate exploratory analysis and randomization testing with MULTIV. Coenoses, v.12, p.145-148, 1997.

PILLAR, V.D.P.; ORLÓCI, L. On randomization testing in vegetation science: multifactor comparisons of relevé groups. Journal of Vegetation Science, v.7, p.585-592, 1996.

ROCHA, M.G. Suplementação a campo de bovinos de corte. In: LOBATO, J.F.P.; BARCELLOS, J.O.J.; KESSLER, A.M. (Eds.) Produção de bovinos de corte. Porto Alegre: Edipucas, 1998. p.77-96.

SIQUEIRA, J.F.; SCHERER, E.E.; TASSINARI, G. et al. Recomendação de adubação e calagem para os estados do Rio Grande do Sul e Santa Catarina. Passo Fundo: 1987. 100p.

SKINNER, R.H.; NELSON, C.J. Effect of tiller trimming on phyllochron and tillering regulation during tall fescue development. Crop Science,v.35, p.4-10, 1995.

TOTHILL, J.C.; HARGREAVES, J.N.G.; JONES, R.M. et al. BOTANAL - A comprehensive sampling and computing procedure for estimating pasture yield and composition. 1. Field sampling. Tropical Agronomy Technical Memorandum, v.78, p.1-24, 1992.

VIÉGAS, J. Análise do desenvolvimento foliar e ajuste de um modelo de previsão do rendimento potencial de matéria seca de L. multiflorum (Lolium multiflorum Lam.). Porto Alegre: Universidade Federal do Rio Grande do Sul, 1998. 159 p. Tese (Doutorado em Zootecnia) - Universidade Federal do Rio Grande do Sul, 1998.

ZARROUGH, K.M.; NELSON, C.J.; SLEPER, D.A. Interrelationship between rates of leaf appearance an tillering in selected tall fescue populations. Crop Science, v.24, p.565-569, 1984.

Recebido em: 28/10/03

Aceito em: 12/08/04 